This is a preprint of: "Different approaches to the global periodicity problem", Anna Cima, Armengol Gasull, Víctor Mañosa, Francesc Mañosas, in Discrete dynamical systems and applications (Proceedings of ICDEA2012), vol. 180, 85-106, Springer Proceedings in Mathematics Statistics, Springer, Berlin, Heidelberg, 2016.

DOI: $\left[10.1007 / 978-3-662-52927-0 \_7\right]$

\title{
Different approaches to the global periodicity problem
}

Anna Cima, Armengol Gasull, Víctor Mañosa, Francesc Mañosas

\begin{abstract}
Let $F$ be a real or complex $n$-dimensional map. It is said that $F$ is $g$ lobally periodic if there exists some $p \in \mathbb{N}^{+}$such that $F^{p}(x)=x$ for all $x$, where $F^{k}=F \circ$ $F^{k-1}, k \geq 2$. The minimal $p$ satisfying this property is called the period of $F$. Given a $m$-dimensional parametric family of maps, say $F_{\lambda}$, a problem of current interest is to determine all the values of $\lambda$ such that $F_{\lambda}$ is globally periodic, together with their corresponding periods. The aim of this paper is to show some techniques that we use to face this question, as well as some recent results that we have obtained. We will focus on proving the equivalence of the problem with the complete integrability of the dynamical system induced by the map $F$, and related issues; on the use of the local linearization given by the Bochner Theorem; and on the use the Normal Form theory. We also present some open questions in this setting.
\end{abstract}

Anna Cima

Departament de Matemàtiques;

Universitat Autònoma de Barcelona, Spain.

e-mail: cima@mat.uab.cat

Armengol Gasull

Departament de Matemàtiques;

Universitat Autònoma de Barcelona, Spain.

e-mail: gasull@mat.uab.cat

Víctor Mañosa

Departament de Matemàtica Aplicada III;

Control, Dynamics and Applications Group,

Universitat Politècnica de Catalunya, Spain.

e-mail: victor.manosa@upc.edu

Francesc Mañosas

Departament de Matemàtiques;

Universitat Autònoma de Barcelona, Spain.

e-mail: manyosas@mat.uab.cat 


\section{Introduction}

This paper deals with globally periodic maps and difference equations. A map $F$ : $\mathscr{U} \rightarrow \mathscr{U}$, defined on an open set $\mathscr{U} \subseteq \mathbb{K}^{n}$ (where $\mathbb{K}$ is either $\mathbb{R}$ or $\mathbb{C}$ ) is globally periodic if there exists some $p \in \mathbb{N}$ such that $F^{p}(x)=x$ for all $x \in \mathscr{U}$, where $F^{k}=$ $F \circ F^{k-1}, k \geq 2$. The minimal $p$ satisfying this property is called the period of $F$.

Observe that if $F$ is globally periodic, then all the orbits of the discrete dynamical system generated by $F$ are periodic.

This paper, rather of being a survey on global periodicity, wants to show the techniques that we have developed to detect globally periodic maps inside a parametric families of maps. The reader is addressed to [3, 28, 36], and the references therein, to have a general overview on the problem.

We start by showing some simple and well-known examples of globally periodic maps:

Example 1. Some linear fractional maps defined in suitable open subsets of $\mathbb{R}^{2}$ or $\mathbb{R}^{3}:$

a) The 5-periodic map $F(x, y)=(y,(1+y) / x)$, is a paradigmatic example known as the Lyness' map. This map is associated to the celebrated difference equation $x_{n+2}=\left(1+x_{n+1}\right) / x_{n}$.

b) The 6-periodic map $F(x, y)=(y, y / x)$.

c) The 8-periodic maps $F^{ \pm}(x, y, z)=(y, z,( \pm 1+y \pm z) / x)$. The plus case is known as the Todd's map. The minus one appears in [20].

Example 2. The piecewise linear map $F(x, y)=(y, \max (0, y)-x)$ is 5-periodic, see [20]. Its associated globally 5-periodic recurrence is

$$
x_{n+2}=\max \left(0, x_{n+1}\right)-x_{n} .
$$

This recurrence is the ultradiscrete version of the Lyness recurrence; see [46] for further details on ultradiscrete systems.

Example 3. The Coxeter's maps (see [18, 23] and also Section 3.3), which are $(n+$ 3)-periodic:

$$
F_{n}\left(x_{1}, \ldots, x_{n}\right)=\left(x_{2}, \ldots, x_{n}, 1-\frac{x_{n}}{\left.1-\frac{x_{n-1}}{1-\frac{x_{n-2}}{1-\cdots \frac{x_{2}}{1-x_{1}}}}\right)}\right.
$$

All of the above maps are associated to some difference equation. Recall that, in general, it is equivalent to study a map of the form

$$
F\left(x_{1}, \ldots, x_{n}\right)=\left(x_{2}, \ldots, x_{n-1}, f\left(x_{1}, \ldots, x_{n}\right)\right),
$$

or the difference equation 


$$
x_{j+n}=f\left(x_{j}, x_{j+1}, \ldots, x_{j+n-1}\right) .
$$

Example 4. The maps

$$
F(x, y)=\left(\frac{y}{1+x+y}, \frac{-x}{1+x+y}\right)
$$

and

$$
F(x, y)=\left(\frac{-y}{\sqrt[3]{1+4 y^{3}-4 x^{3}}}, \frac{x}{\sqrt[3]{1+4 y^{3}-4 x^{3}}}\right)
$$

are 4-periodic. These maps are given by the flow at time $2 \pi / 4$ of the planar $2 \pi$ isochronous centers $\dot{x}=-y+x^{2}, \dot{y}=x(1+y)$, and $\dot{x}=-y+4 x^{2} y^{2}, \dot{y}=x+4 x y^{3}$, respectively. Recall that a differential system, or a vector field, is called isochronous in an invariant open set $\mathscr{U} \subset \mathbb{R}^{n}$, if there exists $T>0$ such that $\varphi(T, \mathbf{x})=\mathbf{x}$ for all $\mathbf{x} \in \mathscr{U}$, where $\varphi$ is the flow associated to the differential system. In this case the flow is also called isochronous. It is clear that for each $p \in \mathbb{N}^{+}$, every $T$-isochronous flow $\varphi$ gives rise to several globally $p$-periodic maps, via the stroboscopic maps $F_{j}(\mathbf{x})=\varphi(j T / p, \mathbf{x})$, where and $j \in \mathbb{N}^{+}, j<p$ and $(j, p)=1$.

Given a $m$-dimensional parametric family of maps, say $F_{\lambda}$, the goal of this work is to determine all the values of $\lambda$ such that $F_{\lambda}$ is globally periodic, together with their corresponding periods. A simple example is given by the family of maps $F(x, y)=(y,(\lambda+y) / x), \lambda \in \mathbb{C}$, which are globally periodic if and only if $\lambda \in\{0,1\}$.

In this paper we will summarize the tools that we are using to face this question. More concretely, the techniques that we will present here are:

- Detect some special properties of the dynamical system induced by globally periodic maps; see Section 2.

- Use the local linearization given by the Bochner Theorem; see Section 3.

- Find integrability type results and relate globally periodic maps with isochronous flows; see Section 4. .

- Use of the Normal Forms Theory to compute some periodicity conditions; see Section 5 .

We will pay a special attention to those families of maps coming from globally periodic difference equations.

\section{General properties of globally periodic maps}

\subsection{Some properties}

From now on, $\mathscr{U}$ will denote an open set of $\mathbb{K}^{n}$, where $\mathbb{K}$ can be $\mathbb{R}$ or $\mathbb{C}$, depending on the context. We list some well-known properties of globally periodic maps that can be used to detect them in some given parametric families of maps. 
(i) If a map $F$ is $p$-periodic, then for any integer $k$ the map $F^{k}$ is also periodic.

(ii) If a map $F: \mathscr{U} \rightarrow \mathscr{U}$ is periodic then it has to be bijective in $\mathscr{U}$. Indeed $F^{-1}=$ $F^{p-1}$.

(iii) Any pointwise periodic homeomorphism in $\mathscr{U}$ must be globally periodic; see [38]. Recall that $F$ is called pointwise periodic in $\mathscr{U}$ if for every $\mathbf{x} \in \mathscr{U}$ there exists an integer $k(\mathbf{x})$ such that $F^{k(\mathbf{x})}(\mathbf{x})=\mathbf{x}$.

(iv) If a rational map is $p$-periodic in an open subset of $\mathbb{K}^{n}$ then it has to be periodic, also of period $p$, in the whole real or complex space, except at the points where $F$ or its iterates are not well defined.

(v) If $F: \mathscr{U} \rightarrow \mathscr{U}$ is a $p$-periodic map and $\mathbf{x} \in \mathscr{U}$ is a fixed point of $F$ then $\left((D F)_{\mathbf{x}}\right)^{p}=\mathrm{Id}$. Thus the eigenvalues of $(D F)_{\mathbf{x}}$ have to be $p$-roots of the unity. Moreover, the matrix $(D F)_{\mathbf{x}}$ diagonalices in $\mathbb{C}$; see [35].

(vi) The fixed points of a periodic map are neither attractors nor repellers.

(vii) Rational globally periodic maps have zero algebraic entropy; see [2],[24].

\subsection{Some applications of the above properties}

In this section we will consider the family of $n$-th order rational difference equation

$$
x_{j+n}=\frac{A_{1} x_{j}+A_{2} x_{j+1}+\cdots+A_{n} x_{j+n-1}+A_{0}}{B_{1} x_{j}+B_{2} x_{j+1}+\cdots+B_{n} j_{j+n-1}+B_{0}},
$$

with initial condition $\left(x_{1}, x_{2}, \ldots, x_{n}\right) \in(0, \infty)^{n}$, and $\sum_{i=0}^{n} A_{i}>0, \sum_{i=0}^{n} B_{i}>0, A_{i} \geq$ $0, B_{i} \geq 0$, and $A_{1}^{2}+B_{1}^{2} \neq 0$.

The following well-known globally periodic difference equations, see Example 1, belong to the above family:

$$
\begin{gathered}
x_{j+2}=\frac{x_{j+1}}{x_{j}}, \quad x_{j+2}=\frac{x_{j+1}+1}{x_{j}}, \quad x_{j+3}=\frac{x_{j+1}+x_{j+2}+1}{x_{j}} \\
x_{j+1}=x_{j}, \quad x_{j+1}=\frac{1}{x_{j}} .
\end{gathered}
$$

The two last ones are known as trivial ones.

Observe that each $p$-periodic $n$-th order difference equation produces in a natural way periodic difference equations of higher order, see [14]. For instance, the following ones of second order:

$$
x_{j+2}=\frac{x_{j+1}}{x_{j}}, \quad x_{j+2}=\frac{x_{j+1}+1}{x_{j}},
$$

give rise to

$$
x_{j+2 \ell}=\frac{x_{j+\ell}}{x_{j}}, \quad x_{j+2 \ell}=\frac{x_{j+\ell}+1}{x_{j}},
$$


for any positive integer $\ell$, which are also periodic. Moreover taking $x_{n}=\alpha y_{n}, \alpha \neq 0$ they can be written as

$$
y_{j+2 \ell}=\frac{\alpha y_{j+\ell}}{y_{j}}, \quad y_{j+2 \ell}=\frac{\alpha y_{j+\ell}+\alpha^{2}}{y_{j}} .
$$

We will say that these difference equations are equivalent to (7).

Next result is obtained by using almost all the properties listed in Section 2.1.

Theorem 1 ([14]) Consider the n-th order rational difference equation (5) with the above mentioned hypotheses. Then, for $n \in\{1,2,3,4,5,7,9,11\}$, any globally periodic recurrence in the family is equivalent to one of the difference equations given in (6).

Open question: Is the above result true for any $n$ ?

Remark 2 If $A_{j}$ and $B_{j}$ are no more non-negative and $n=1$, then there are globally periodic recurrences of type (5) of all periods: the Möbius transformations.

Remark 3 When $n=2$, that is, for difference equations of the form,

$$
x_{j+2}=\frac{A_{1} x_{j}+A_{2} x_{j+1}+A_{0}}{B_{1} x_{j}+B_{2} x_{j+1}+B_{0}}, \quad A_{i}, B_{i} \in \mathbb{C},
$$

by using the property that the globally periodic maps have zero algebraic entropy, it has been proved in [6, 7] that the only possible periods for the globally periodic cases are 6, 5, 8, 12, 18, and 30. Moreover all them are realizable; see also [42, 43]. Again new cases, non-equivalent to the ones of the list given in (6), appear.

\subsection{A remark about reversibility}

Traditionally a map $F$ is said to be reversible if $F=\sigma_{1} \circ \sigma_{2}$ where $\sigma_{1}, \sigma_{2}$ are involutions. Curiously all explicit known globally periodic recurrences have an associated map $F: U \rightarrow U$, where $U$ is a open subset of $\mathbb{R}^{n}$, that satisfies that $\sigma \circ F$ is an involution where $\sigma: \mathbb{R}^{n} \rightarrow \mathbb{R}^{n}$ is defined by $\sigma\left(x_{1}, x_{2}, \ldots, x_{n-1}, x_{n}\right)=\left(x_{n}, x_{n-1}, \ldots, x_{2}, x_{1}\right)$. Then for these periodic recurrences we get $F=\sigma \circ(\sigma \circ F)$ and they are reversible.

Due to this fact some authors claimed that this is a general property for the real recurrences and tried to prove it. The following implicit example shows that there are recurrences $F$ such that $\sigma \circ F$ is not an involution.

Proposition 4 ([15]) There exists a second order globally 3-periodic recurrence which associated map $F$ is such that $\sigma \circ F$ is not an involution.

Proof. Consider the map $F=\Phi \circ L \circ \Phi^{-1}$, where 


$$
\Phi(x, y)=(x+g(y), y+g(-x-y))
$$

with

$$
g(z)=-z-z^{2}-z^{3} \quad \text { and } \quad L(x, y)=(y,-x-y) .
$$

It can be seen that $F$ writes as $F(x, y)=(y, f(x, y))$ for a suitable $f$. Since $F$ is 3-periodic we get that $F^{-1}(x, y)=(f(x, y), x)$. On the other hand $(\sigma \circ F \circ \sigma)(x, y)=$ $(f(y, x), x)$. Then the equality $\sigma \circ F \circ \sigma=F^{-1}$ which is equivalent to $(\sigma \circ F)^{2}=\mathrm{Id}$ is also equivalent to $f(x, y)=f(y, x)$. Computing the Taylor series of $f$ we obtain $f(x, y) \neq f(y, x)$. Hence $\sigma \circ F$ is not an involution as we wanted to prove.

Nevertheless observe that for the above map $F=\Phi \circ L \circ \Phi^{-1}$, if we consider $\widetilde{\sigma}:=\Phi \circ \sigma \circ \Phi^{-1}$ it holds that:

- $\tilde{\sigma}$ is an involution.

- The map $\widetilde{\sigma} \circ F$ is also an involution.

So the following natural question arises:

Open question: Is any globally periodic recurrence reversible?

\section{Linearization of globally periodic maps and recurrences}

\subsection{The Bochner Theorem}

Let $\mathscr{V}$ be an open subset homeomorphic to $\mathbb{K}^{n}$. It is said that a map $F: \mathscr{V} \rightarrow \mathscr{V}, \mathscr{C}^{r}$ linearizes (globally) if there exists a $\mathscr{C}^{r}$-diffeomorphism, $\psi$, with $r \in\{0,1, \ldots, \infty, \omega\}$, such that $\psi \circ F=L \circ \psi$ for all points in $\mathscr{V}$, where $L$ is a linear map defined on a suitable domain.

An adaptation of a classical result due S. Bochner states that every globally periodic map with a fixed point locally linearizes:

Theorem 5 (Bochner [8] and [39, Chap. V]) Let $F: \mathscr{U} \rightarrow \mathscr{U}$ be a p-periodic $\mathscr{C}^{r}$ diffeomorphism, where $\mathscr{U}$ is an open set of $\mathbb{K}^{k}$ and $r \neq 0$. Let $\mathbf{x}_{0} \in \mathscr{U}$ be a fixed point of $F$. Then, there exists a neighbourhood of $\mathbf{x}_{0}$ where $F$ is $\mathscr{C}^{r}$-conjugated with the linear map $L(\mathbf{x})=(D F)_{\mathbf{x}_{0}} \mathbf{x}$. Moreover the linearization is given by the local $\mathscr{C}^{r}$-diffeomorphism

$$
\psi(\mathbf{x})=\frac{1}{p} \sum_{i=0}^{p-1}(D F)_{\mathbf{x}_{0}}^{-i}\left(F^{i}(\mathbf{x})\right) .
$$

Proof. Since $F$ is $p$-periodic $\left((D F)_{\mathbf{x}_{0}}\right)^{p}=\mathrm{Id}$. So $\left(\operatorname{det}\left((D F)_{\mathbf{x}_{0}}\right)^{p}=1\right.$ and $(D F)_{\mathbf{x}_{0}}$ is invertible. Consider $\psi$ as in the statement. By the inverse function theorem it is 
clear that the map $\psi$ is a local $\mathscr{C}^{r}$-diffeomophism because $D \psi\left(\mathbf{x}_{0}\right)=$ Id. Using the $p$-periodicity of $F$ and $(D F)_{\mathbf{x}_{0}}$ we get that for any $\mathbf{x}$ in a certain open neighborhood of $\mathbf{x}_{0}$,

$$
\begin{aligned}
\psi(F(\mathbf{x})) & =\frac{1}{p}\left[F(\mathbf{x})+(D F)_{\mathbf{x}_{0}}^{-1} F^{2}(\mathbf{x})+\cdots+(D F)_{\mathbf{x}_{0}}^{-(p-1)} F^{p}(\mathbf{x})\right] \\
& =\frac{1}{p}(D F)_{\mathbf{x}_{0}}\left[(D F)_{\mathbf{x}_{0}}^{-1} F(\mathbf{x})+\cdots+(D F)_{\mathbf{x}_{0}}^{-p} F^{p}(\mathbf{x})\right] \\
& =(D F)_{\mathbf{x}_{0}} \cdot \psi(\mathbf{x})=L(\psi(\mathbf{x}))
\end{aligned}
$$

and the result follows.

An easy, but useful, consequence of the Bochner Theorem is:

Proposition 6 Let $F$ be a analytic map having a fixed point $\mathbf{x}_{0}$. Assume that $F$ is globally p-periodic and let $k$ be the minimum positive $k$ such that $\left.\left((D F)_{\mathbf{x}_{0}}\right)\right)^{k}=\mathrm{Id}$, then $k=p$.

For instance, a simple corollary of the above result says that if an analytic map $F(x, y)=\left(-x+O_{2}(x, y),-y+O_{2}(x, y)\right)$ is globally $p$-periodic then it has to be an involution, that is $p=2$. Here $O_{2}(x, y)$ denotes second order terms at the origin.

In $\mathbb{R}$ and $\mathbb{R}^{2}$ every $p$-periodic map globally linearize. The last case is a consequence of Kerékjártó's Theorem.

Theorem 7 (Kerékjártó 1919, [19]) Let $\mathscr{U} \subseteq \mathbb{R}^{2}$ be homeomorphic to $\mathbb{R}^{2}$, and let $F$ be a p-periodic map in $\mathscr{U}$. Then $F$ is $\mathscr{C}^{0}$-linearizable.

As a consequence of the above result it is easy to prove that every planar globally periodic map is given by the stroboscopic map of $\mathscr{C}^{0}$ flow.

Corollary 8 For every preserving orientation globally periodic map $F$ defined in an open set $\mathscr{U}$, homeomorphic to $\mathbb{R}^{2}$, there exists a continuous isochronous periodic flow $\varphi$ such that $F(\mathbf{x})=\varphi(T, \mathbf{x})$ for all $\mathbf{x} \in \mathscr{U}$.

Proof. Kerékjártó's Theorem ensures the existence of a homeomorphism $\psi$ such that

$$
\psi \circ F=L \circ \psi
$$

where $L$ is a periodic linear map. On the other hand, we can consider that

$$
L(\mathbf{x})=A \mathbf{x}, \text { with } A=\left(\begin{array}{rr}
\cos T & -\sin T \\
\sin T & \cos T
\end{array}\right),
$$

and where $T=2 k \pi / n,(k, n)=1$ and $n$ is the period of $F$. Hence $L$ is the "time- $T$ " stroboscopic map of the isochronous flow $\varphi_{L}$ associated to the harmonic oscillator $\dot{u}=-v, \dot{v}=u$. Therefore

$$
F(x)=\psi^{-1} \circ L \circ \psi(x)=\psi^{-1} \circ \varphi_{L}(T, \psi(x))=: \varphi(T, x) .
$$


As we will see in next sections, sometimes the Bochner Theorem can be used to construct global linearizations of smooth globally periodic maps. However there are examples of globally periodic homeomorphisms which do not linearize. The first one was given by Bing in 1952, [5, p. 361]; see also [4]. In general, even in a differentiable scenario the search of linearizations for globally periodic maps needs stronger tools than the Bochner Theorem because although any periodic $\mathscr{C}^{1}$ diffeomorphism in $\mathbb{R}^{k}$, with $k \leq 6$ must have a fixed point, there are periodic $\mathscr{C}^{1}$ diffeomorphisms in $\mathbb{R}^{k}, k \geq 7$ without fixed points; see [32,34].

\subsection{Applications of the Bochner theorem}

Some results that can be proved using Bochner Theorem are the following:

Theorem 9 ([16]) Any $(n+1)$-periodic recurrence of class $\mathscr{C}^{k}$ defined in an open connected subset of $\mathbb{R}^{n}$ can be $\mathscr{C}^{k}$-linearized.

The proof consists in showing that the Bochner linearization is in this case globally invertible. For other globally $p$-periodic difference equations the same idea also works.

Unfortunately, recently we have proved that there are simple explicit involutions $(p=2)$, not coming from a difference equation, for which the linearization given by the Bochner Theorem is not globally invertible; see [17]. Non explicit examples appear in [45].

Proposition 10 ([17]) Let $F: \mathbb{R}^{2} \longrightarrow \mathbb{R}^{2}$ be given by

$$
F(x, y)=\left(x+4 x y+f(x, y),-y+2\left(x^{2}+y^{2}\right)-f(x, y)\right),
$$

where $f(x, y)=4(x+y)^{2}(y-x)-4(x+y)^{4}$. Then $F$ is an involution, has $(0,0)$ as a fixed point and its associated Bochner linearization $\psi=\frac{1}{2}\left(\operatorname{Id}+(D F)_{(0,0)}^{-1} \circ F\right)$ is not a global diffeomorphism.

Despite this example, the global invertibility of the Bochner linearizations for maps coming from difference equations is still an open question.

\section{Open questions:}

Let $\mathscr{U}$ be an open set, homeomorphic to $\mathbb{R}^{n}$.

- Assume that $\mathscr{U}$ is invariant by the map associated to a globally periodic $\mathscr{C}^{1}$-difference equation. Is the Bochner linearization associated to a fixed point in $\mathscr{U}$ globally invertible?

- Is any globally periodic $\mathscr{C}^{1}$-map, $F: \mathscr{U} \rightarrow \mathscr{U}$ globally linearizable? 


\subsection{A fascinating example: the Coxeter recurrence}

Notice that the global linearizations are not only associated to a given globally periodic difference equation but also to a given invariant open set, homeomorphic to $\mathbb{R}^{n}$. For instance, consider the 5-periodic Lyness' map $F(x, y)=(y,(1+y) / x)$. It can be seen that $F$ and its iterates are defined in $\mathbb{R}^{2} \backslash \mathscr{L}$ where $\mathscr{L}$ is the union of the straight lines $x=0, x=-1, y=0, y=-1, x+y=-1$. Clearly $\mathbb{R}^{2} \backslash \mathscr{L}$ has twelve connected components and $F$ fixes two of them and permutes the rest. We denote by $\mathscr{U}_{1}=\left\{(x, y) \in \mathbb{R}^{2}: x>0, y>0\right\}$ and by $\mathscr{U}_{2}$ the interior of the triangle with vertices $(-1,0),(-1,-1)$ and $(0,-1)$ which are the two invariant components of $F$. By using the Bochner theorem we prove:

Proposition 11 ([16]) The maps $\left.F\right|_{\mathscr{U}_{1}}$ and $\left.F\right|_{\mathscr{U}_{2}}$ are globally $\mathscr{C}^{\omega}$-linearizable.

The Coxeter recurrence, which is an extension of the Lyness one, is much more complicated; see [18]. For instance, Coxeter maps have many fixed points and on a neighborhood of each of them they are locally conjugate with different linear models.

In the statement of the two following results [ ] denotes the integer part function.

Lemma 12 ([18]) There are exactly $[(n+2) / 2]$ linear globally periodic recurrences of order $n$, and period $n+3$ without a line of fixed points (that is without eigenvalue $\lambda=1)$.

Theorem 13 ([18]) The Coxeter map $F_{n}$ associated to the recurrence (2), has exactly $[(n+2) / 2]$ fixed points. Moreover at each of these fixed points $F_{n}$ is locally conjugated to a different linear map.

In other words all linear $(n+3)$-periodic recurrence of order $n$ without the eigenvalue 1 , is present in the Coxeter map $F_{n}$.

It would be interesting to know if the corresponding local conjugations given by the Bochner Theorem are global in the corresponding invariant open sets.

\section{Integrability properties of globally periodic maps}

\subsection{Existence and construction of complete set of first integrals}

A non-constant function $V: \mathscr{U} \subseteq \mathbb{R}^{n} \rightarrow \mathbb{R}$ is called a first integral or invariant of the dynamical system generated by $F$ if

$$
V(F(\mathbf{x}))=V(\mathbf{x})
$$

If a map $F$ possesses a first integral $V$, then all the orbits lie in some level set of $V$, or in other words, the level sets of $V$ are invariant by $F$. 
A map $F: \mathscr{U} \subseteq \mathbb{R}^{n} \rightarrow \mathbb{R}^{n}$ is integrable if it has $n-1$ functionally independent first integrals in $\mathscr{U}$, and completely integrable if it has $n$ functionally independent first integrals in $\mathscr{U}$. In [10], it was proved that there is a strong relation between globally periodic maps and complete integrability.

Theorem 14 ([10]) Let $F: \mathscr{U} \subseteq \mathbb{R}^{n} \rightarrow \mathscr{U}$ be an injective analytic map defined in an open set $\mathscr{U}$. The following statements hold:

(i) If $F$ is globally periodic, then it is completely integrable.

(ii) If $F$ is completely integrable and it has $n$ functionally independent first integrals $V_{1}, V_{2}, \cdots, V_{n}$ such that

$$
\operatorname{Card}\left(\bigcap_{i=1}^{n}\left\{V_{i}=c_{i}\right\} \cap \mathscr{U}\right) \leq K, \text { for all } c_{i} \in \mathbb{R},
$$

being $K$ a given positive integer, then $F$ is globally periodic.

Some remarks:

1. The proof of this result is constructive, so given a globally periodic map $F$, we are able to obtain a complete set of $n$ first integrals; see Proposition 15.

2. Notice that the statement is given for analytic maps because we use strongly this condition to prove that the system of first integrals that we construct is formed by $n$ functionally independent functions. However in many cases the method of construction gives functionally independent integrals even if the regularity is relaxed. For instance in [10, Prop. 18] two functionally independent integrals are given for the continuous map associated to the recurrence (1).

3. Condition (9) cannot be removed as the following example shows: Consider the $\operatorname{map} F: \mathbb{R}^{2} \rightarrow \mathbb{R}^{2}$

$$
F(x, y)=(x+2 \pi, y) .
$$

This map $F$ is bijective on the whole $\mathbb{R}^{2}$ and it is clearly not $p$-periodic for all $p \in \mathbb{N}$. Moreover, the dynamical system generated by it is completely integrable. For instance, $V_{1}(x, y)=y-\sin (x)$ and $V_{2}(x, y)=y$ are two functionally independent first integrals for $F$. On the other hand, it is clear that for many $c_{1}$ and $c_{2}$, the finiteness condition (9) is not satisfied.

Example 5. It is well known that the Lyness map $F(x, y)=(y,(a+y) / x)$ has, for all value $a \in \mathbb{C}$, the first integral

$$
V_{1, a}(x, y)=\frac{(x+1)(y+1)(x+y+a)}{x y} .
$$

When $a=0$ it has also the first integral

$$
V_{2,0}(x, y)=\frac{x^{4} y^{2}+x^{2} y^{4}+y^{4}+y^{2}+x^{2}+x^{4}}{x^{2} y^{2}}
$$

and for $a=1$, the first integral $V_{2,1}(x, y)=P(x, y) /\left(x^{2} y^{2}\right)$, where 


$$
\begin{aligned}
P(x, y)= & x y^{4}+\left(x^{3}+x^{2}+2 x+1\right) y^{3}+\left(x^{3}+5 x^{2}+3 x+2\right) y^{2} \\
& +\left(x^{4}+2 x^{3}+3 x^{2}+3 x+1\right) y+x^{3}+2 x^{2}+x .
\end{aligned}
$$

It can be easily seen that $V_{1, i}$ and $V_{2, i}$ for $i=0$ or $i=1$ are couples of functionally independent functions. So, as Theorem 14 predicts, globally periodic cases are completely integrable.

We also recall how to construct many independent first integrals for globally periodic maps.

Proposition 15 ([10]) Let $F: \mathscr{U} \subset \mathbb{R}^{n} \rightarrow \mathscr{U}$ be a globally p-periodic map on $U$. Let

$$
\Phi: \mathscr{U}^{p}=\overbrace{\mathscr{U} \times \mathscr{U} \times \cdots \times \mathscr{U}}^{p} \longrightarrow \mathbb{K}
$$

be a symmetric function. Then, whenever it is not a constant function,

$$
V_{\phi}(\mathbf{x})=\Phi\left(\mathbf{x}, F(\mathbf{x}), \ldots, F^{p-1}(\mathbf{x})\right)
$$

is a first integral of the dynamical system generated by $F$.

Using the above result with some families of symmetric functions, for instance symmetric polynomials, it is possible to obtain complete sets of first integrals, see again [10].

Example 6. Consider the map $F(x, y)=(y, c /(x y))$, corresponding to the difference equation $x_{n+2}=c /\left(x_{n} x_{n+1}\right)$. It holds that

$$
(x, y) \rightarrow\left(y, \frac{c}{x y}\right) \rightarrow\left(\frac{c}{x y}, x\right) \rightarrow(x, y),
$$

so $F$ is globally 3-periodic. In this case, then $n=2$ and $p=3$.

A complete set of first integrals can be constructed using two suitable symmetric functions. Taking $\sigma_{1}(a, b, c, d, e, f)=a+b+c+d+e+f$, we get:

$$
V_{1}(x, y)=2\left(x+y+\frac{c}{x y}\right) .
$$

Taking now $\sigma_{2}(a, b, c, d, e, f)=a^{2}+b^{2}+c^{2}+d^{2}+e^{2}+f^{2}$, we obtain

$$
V_{2}(x, y)=2\left(x^{2}+y^{2}+\frac{c^{2}}{x^{2} y^{2}}\right) .
$$

Note that the symmetric function $\sigma_{3}(a, b, c, d, e, f)=a b c d e f$ gives a constant function and is not a first integral. It is easy to prove that $V_{1}$ and $V_{2}$ are functionally independent first integrals.

Example 7. Using the symmetric function $S_{2}\left(a_{1}, \ldots, a_{n p}\right)=a_{1}^{2}+a_{2}^{2}+\ldots+a_{n p}^{2}$, we get that any globally $p$-periodic map $F: \mathscr{U} \subset \mathbb{R}^{n} \rightarrow \mathscr{U}$ has always the first integral 


$$
V(\mathbf{x})=\sum_{i=0}^{p-1}\left\|F^{i}(\mathbf{x})\right\|^{2} .
$$

It is easy to see that the non-empty level sets of this first integral are compact. This will be usefull in the proof of Proposition 22.

\subsection{A remark on invariant curves of rational maps and global periodicity}

Globally periodic rational maps in $\mathbb{K}^{2}$ are in fact birational (i.e. its inverse is also rational), because $F^{-1}=F^{p-1}$. By Theorem 14 and Proposition 15 in this case a complete set of rational first integrals can be chosen. Consequently, they preserve a couple of fibrations of the plane, which are generically transversal, and given by algebraic curves.

A theorem of Hurwitz of 1893, states that any compact Riemann surface with genus $g>1$ admits at most $84(g-1)$ conformal automorphisms, that is, homeomorphisms of the surface onto itself which preserve the local structure; see [21, 22]. In our context, Hurwitz theorem can be stated as (see [33]):

Theorem 16 (Hurwitz, 1893) The group of birational maps on a non-singular algebraic curve of genus $g>1$ is finite of order at most $84(g-1)$.

The following result, is a consequence of the above one and properties (iii) and (iv) given in Section 2.1.

Corollary 17 A birational map in $\mathscr{U} \subseteq \mathbb{K}^{2}$ with a rational first integral $V$, such that the curves $\{V=c\}$ have generically genus $g>1$, must be globally periodic.

Proof. If the curves $\{V=c\}$ have generically genus $g>1$ then there exists an open set $\mathscr{V} \subseteq \mathscr{U}$ foliated by curves of these type. By Hurwitz Theorem on each of these curves the map must be periodic, so $F$ is pointwise periodic on $\mathscr{V}$, and hence by the Montgomery Theorem ([38], see also property (iii) in Section 2.1) $F$ must be globally periodic on $\mathscr{V}$. Since $F$ is rational then it must be periodic on the whole $\mathbb{K}^{2}$ except at the points where its iterates are not well defined, as property $(i v)$ in Section 2.1, states.

Example 8. It is now common knowledge that for the Lyness map $F(x, y)=(y$, $(a+y) / x)$, and for all value $a \in \mathbb{C}$ the first integrals $V_{1, a}$ given in Example 5 produce fibrations of the plane given by curves that have generically genus 1 (of course, for some particular level sets the curves are straight lines so they have genus 0 ).

It is interesting to notice that when $a=0$ the integral $V_{2,0}$ produces fibrations of the plane given by curves that have generically genus 7 . Finally, when $a=1$ the integral $V_{2,1}$ generically fibers the plane by curves of genus 6 . 


\subsection{Measure preservation}

Another interesting property is that any globally periodic map has associated a natural invariant measure. Recall that a measure $m$ is invariant for a map $F$ if $m\left(F^{-1}(B)\right)=m(B)$ for any measurable set $B$.

Lemma 18 Let $\mathscr{U}$ be an open subset of $\mathbb{R}^{n}$ and let $F: \mathscr{U} \rightarrow \mathscr{U}$ be a $\mathscr{C}^{k}$-map globally periodic map, $k \geq 1$. Then $F$ has the invariant measure

$$
m(B)=\sum_{i=0}^{p-1} \int_{F^{i}(B)} d \mathbf{x} .
$$

Moreover $m$ is absolutely continuous with respect the Lebesgue measure with $\mathscr{C}^{k-1}$. density $v(\mathbf{x})=\sum_{i=0}^{p-1}\left|\operatorname{det}\left(D F^{i}(\mathbf{x})\right)\right|$.

Proof. Let $B$ be a Lebesgue measurable subset of $\mathscr{U}$. Since $F^{-1}=F^{p-1}$,

$$
m\left(F^{-1}(B)\right)=\sum_{i=0}^{p-2} \int_{F^{i}(B)} d \mathbf{x}+\int_{F^{-1}(B)} d \mathbf{x}=\sum_{i=0}^{p-1} \int_{F^{i}(B)} d \mathbf{x}=m(B) .
$$

So $m$ is an invariant measure.

By using the change of variables formula we obtain

$$
m(B)=\sum_{i=0}^{p-1} \int_{F^{i}(B)} d \mathbf{x}=\int_{B} \sum_{i=0}^{p-1}\left|\operatorname{det}\left(D F^{i}(\mathbf{x})\right)\right| d \mathbf{x}=\int_{B} v(\mathbf{x}) d \mathbf{x},
$$

giving the desired result. Notice that $v$ is a $\mathscr{C}^{k-1}$ function because the global periodicity of $F$ implies that $\operatorname{det}\left(D F^{i}(\mathbf{x})\right) \neq 0$.

\subsection{Existence and construction of Lie Symmetries}

A Lie Symmetry of a map $\mathrm{F}$ is a vector field $X$ such that $F$ maps any orbit of the differential system

$$
\dot{\mathbf{x}}=X(\mathbf{x}),
$$

into another orbit of the system. An equivalent definition is the following: It is a vector field $\mathbf{X}$ such that the differential equation (10) is invariant by the change of variables $\mathbf{u}=F(\mathbf{x})$. This property is characterized by

$$
X(F(\mathbf{x}))=D F(\mathbf{x}) X(\mathbf{x}), \quad \text { for all } \quad \mathbf{x} \in \mathscr{U} .
$$

The existence of Lie Symmetries is an important issue in the theory of discrete integrability; see for instance [30]. From a dynamic viewpoint this importance is 
clear in the integrable case, where the dynamics are in fact one dimensional. Next results illustrate this fact.

Theorem 19 ([11]) Let $F: \mathscr{U} \subseteq \mathbb{R}^{n} \rightarrow \mathscr{U}$ be a diffeomorphism having a Lie symmetry $\mathbf{X}$, and let $\gamma$ be an orbit of $X$, preserved by $F$, i.e. $F: \gamma \rightarrow \gamma$. Then the dynamics of $F$ restricted to $\gamma$ is either conjugated to a rotation, conjugated to a translation of the line, or constant, according whether $\gamma$ is homeomorphic to $\mathbb{S}^{1}, \mathbb{R}$, or a point, respectively.

Theorem 20 ([11]) Let $F$ be an integrable map, with functionally independent first integrals $V_{1}, \ldots, V_{n-1}$ and such that it preserves a measure $m(B)=\int_{B} v(\mathbf{x}) d \mathbf{x}$, with $v \in \mathscr{C}^{1}(\mathscr{U})$ and non-vanishing in $\mathscr{U}$. Then $F$ has a Lie Symmetry $X$ sharing with $F$ the same set of functionally independent first integrals. Moreover

$$
\begin{aligned}
& X(\mathbf{x})=\left(-\partial V_{1}(\mathbf{x}) / \partial y, \partial V_{1}(\mathbf{x}) / \partial x\right) / v(\mathbf{x}) \text { if } n=2, \text { and } \\
& X(\mathbf{x})=\left(\nabla V_{1}(\mathbf{x}) \times \nabla V_{2}(\mathbf{x}) \times \cdots \times \nabla V_{n-1}(\mathbf{x})\right) / v(\mathbf{x}) \text { if } n>2,
\end{aligned}
$$

where $\times$ denotes the cross product in $\mathbb{R}^{n}$.

Summarizing the results of this section we have:

1. Globally periodic maps have $n$ functionally independent first integrals.

2. Every globally periodic map has an invariant measure with density $v(\mathbf{x})=$ $\sum_{i=0}^{p-1}\left|\operatorname{det}\left(D F^{i}(\mathbf{x})\right)\right|$.

3. An integrable map with an invariant measure $m(B)=\int_{B} v(\mathbf{x}) d \mathbf{x}$, has a Lie Symmetry of the form (12).

Collecting all the facts above, we have that every globally periodic map has $n$ linearly independent Lie Symmetries, thus obtaining the following result:

Corollary 21 Let $F$ be a globally p-periodic map defined on an open set $\mathscr{U} \subseteq \mathbb{R}^{n}$, with a complete set of first integrals $V_{1}, \ldots, V_{n}$. Then there exist $n$ Lie Symmetries of $F$ given by

$$
X_{k}(\mathbf{x})=\left(-\partial V_{k}(\mathbf{x}) / \partial y, \partial V_{k}(\mathbf{x}) / \partial x\right) / v(\mathbf{x}),
$$

for $k=1,2$, if $n=2$ and

$$
X_{k}(\mathbf{x})=\left(\nabla V_{1}(\mathbf{x}) \times \nabla V_{2}(\mathbf{x}) \times \cdots \times \widehat{\nabla V_{k}(\mathbf{x})} \times \cdots \times \nabla V_{n}(\mathbf{x})\right) / v(\mathbf{x}),
$$

for all $k=1, \ldots, n$, if $n>2$. In all cases $v$ can be chosen as in Lemma 18.

We will say that a flow of a differential equation is periodic if all their trajectories are either periodic or critical points. Next result shows that at least $n-1$ of the above Lie Symmetries give rise to periodic flows as a consequence of the result below.

Proposition 22 Let $F: \mathscr{U} \subseteq \mathbb{R}^{n} \rightarrow \mathbb{R}^{n}$ be a p-periodic map defined in an open set $\mathscr{U}$, having $n-1$ functionally independent first integrals, $V_{1}, \ldots, V_{n-1}$ and being

$$
V(\mathbf{x})=\sum_{i=0}^{p-1}\left\|F^{i}(\mathbf{x})\right\|^{2} .
$$


the first integral given in Example 7. Then, the induced foliation of $\mathscr{U}$ given by the transversal levels sets $L_{h_{1}, \ldots, h_{n-1}}:=\pitchfork_{i=1}^{n-1}\left\{V_{i}=h_{i}\right\}$ is diffeomorphic to a finite disjoint union of $\mathbb{S}^{1}$.

Proof. Is easy to prove that the nonempty level sets $L_{h_{1}}=\left\{V_{1}=h_{1}\right\}$ are compact. Now, since $V_{1}, \ldots V_{n-1}$ are functionally independent, the locus where they intersect transversally

$$
L_{h_{1}, \ldots, h_{n-1}}:=\left\{\mathbf{x} \in \bigcap_{i=1}^{n-1}\left\{V_{i}=h_{i}\right\} \text { such that } \operatorname{Rank}\left(\nabla V_{1}(\mathbf{x}), \ldots, \nabla V_{n-1}(\mathbf{x})\right)=n-1\right\}
$$

is a compact 1-dimensional manifold, so it must be diffeomorphic to a finite disjoint union of $\mathbb{S}^{1}$; see for instance [29, p. 208].

Therefore, the associated Lie symmetries give rise to periodic orbits on each connected component of the (non-empty) sets $L_{h_{1}, \ldots, h_{n-1}}$ which are, of course, generic. Also observe that the locus of non-transversality of the energy levels will correspond to singular points of the associated Lie Symmetries. Therefore there exist at least $n-1$ linearly independent periodic Lie symmetries.

\section{Open question:}

- Is every orientation preserving globally periodic map of class $\mathscr{C}^{1}$ associated to a periodic flow $\varphi$ ? That is: Is it true that

$$
F(\mathbf{x})=\varphi(\tau(\mathbf{x}), \mathbf{x})
$$

for some periodic flow $\varphi(t, \mathbf{x})$ ?

- Furthermore, is every orientation preserving globally periodic map of class $\mathscr{C}^{1}$ the stroboscopic map of an isochronous flow? That is: Is it true that

$$
F(\mathbf{x})=\varphi(\tau, \mathbf{x})
$$

for some continuous periodic flow $\varphi(t, \mathbf{x})$ and some $\tau \in \mathbb{R}^{+}$?

Observe that the above questions are not equivalent. Indeed, if we have a periodic vector field $X: \mathscr{U} \subseteq \mathbb{R}^{n} \rightarrow \mathbb{R}^{n}$, it can be reparameterized by

$$
Y(\mathbf{x})=T(\mathbf{x}) X(\mathbf{x}):
$$

where $T(\mathbf{x})$ is the function that assigns to each point the period of the periodic orbit passing through it. If $T$ were a smooth function in $\mathscr{U}$ then it would be an 1 -isochronous vector field. But sometimes, $T$ is not regular in $\mathscr{U}$ because it tends to infinity. A characterization of when $T$ is regular has been given recently in [37]. Notice that indeed, there are examples of periodic flows with some periodic orbits 
such that the periods of the neighbouring orbits tends to infinity as they approach to the singular periodic orbit. These examples were constructed by G. Reeb (1952), D. Epstein (1972), D. Sullivan (1976) and E. Voght (1977); see [41, 25, 44, 47] respectively. All these classical examples are not polynomial. Recently we have learned about the existence of a polynomial one due to D. Peralta-Salas [40].

Observe also that the obstruction to give a positive answer to the first question in the above open problem is that, although a globally periodic map has at least $n-1$ Lie Symmetries that are periodic vector fields, it is difficult to guarantee that there exists at least one of them such that $F$ preserves any solution $\gamma$ of the differential equation $\dot{\mathbf{x}}=X(\mathbf{x})$. The following example shows that sometimes this obstruction can be avoided.

Example 9. The map

$$
F(x, y, z)=\left(z, \frac{1+y+z}{x}, \frac{1+x+y+z+x z}{x y}\right)
$$

is globally 4-periodic because it corresponds to $G \circ G$, where $G$ is the Todd's map given in Example 1; see also [12]. In the first octant it has the Lie symmetry given by

$$
X(x, y, z)=x y z\left(\nabla V_{1}(x, y, z) \times \nabla V_{2}(x, y, z)\right)
$$

where

$$
V_{1}=\frac{(x+1)(y+1)(z+1)(1+x+y+z)}{x y z}, \quad V_{2}=\frac{(x+1)(z+1)}{y} .
$$

Since $F$ preserves any solution $\gamma$ of the differential equation $\dot{\mathbf{x}}=X(\mathbf{x})$, this implies that in the first quadrant $F$ can be seen as the stroboscopic map of the isochronous flow of the vector field $Y(\mathbf{x})=T(\mathbf{x}) X(\mathbf{x})$ on $\mathbb{R}^{3}$.

\section{A Normal Form Theory approach}

The theory of Normal forms for dynamical systems, both discrete and continuous, goes back to Poincaré and Lyapunov, and it is a well-known tool for the study of bifurcations of dynamical systems; see for instance [1, 27, 48].

In this section we outline a method, introduced in [13], which is the discrete analog of the way in that Normal Form Theory is applied in the theory of planar differential equations in order to obtain center and isochronicity conditions [9, 26, 31]. First, let us recall briefly how Normal Form theory works for these two problems. Given a planar analytic differential equation

$$
\begin{aligned}
& \dot{x}=-y+f_{2}(x, y)+f_{3}(x, y)+O_{4}(x, y), \\
& \dot{y}=x+g_{2}(x, y)+g_{3}(x, y)+O_{4}(x, y),
\end{aligned}
$$


it is well known that the origin is either a center or a focus. In this later case the stability of the origin is given by the sign of the first non-null number of a list of polynomial expressions in the coefficients of $f_{j}$ and $g_{j}$, called the Lyapunov quantities $V_{3}, V_{5}, \ldots$ obtained from the coefficients of $f_{j}$ and $g_{j}$. In particular, the first Lyapunov quantity only depends on $f_{2}, f_{3}, g_{2}$, and $g_{3}$, that is $V_{3}=V_{3}\left(f_{2}, f_{3}, g_{2}, g_{3}\right)$.

The normal form of (14) is

$$
\begin{aligned}
& \dot{x}=-y-\left(x^{2}+y^{2}\right)\left(V_{3} y+T_{2} x\right)+O_{4}(x, y), \\
& \dot{y}=x+\left(x^{2}+y^{2}\right)\left(V_{3} x-T_{2} y\right)+O_{4}(x, y),
\end{aligned}
$$

see $[27$, p. 154$]$ or $[48$, Sec. $3.1 B]$. So only the term $V_{3}$ (which gives the stability) and the term $T_{2}$ (that gives information about the period of the orbits) remain.

Following the same idea, and since the Bochner Theorem ensures that globally periodic maps with a fixed point locally linearize, we can apply the Normal Form theory to analytic maps with a fixed point in order to obtain some explicit expressions of the obstructions to be locally linearized around the point. The vanishing of these expressions will be necessary conditions for global periodicity.

\subsection{Normal Form Theory and global periodicity}

In this section we briefly recall some issues of Normal Form Theory. We also indicate how to use it to obtain some necessary conditions for global periodicity.

Let $F:=F^{(1)}: \mathbb{K}^{2} \rightarrow \mathbb{K}^{2}$, be a family of smooth maps satisfying $F^{(1)}(0)=0$. Let

$$
F^{(1)}(\mathbf{x})=F_{1}^{(1)}(\mathbf{x})+F_{2}^{(1)}(\mathbf{x})+\cdots+F_{k}^{(1)}(\mathbf{x})+O\left(|\mathbf{x}|^{k+1}\right)
$$

be the Taylor expansion of $F$ at $\mathbf{0}$, where $F_{r}^{(1)} \in \mathscr{H}_{r}$, the real vector space of maps whose components are homogeneous polynomials of degree $r$.

A sequence of transformations $\Phi_{n}$ can be constructed, in such a way that at each step, $\Phi_{n}$ removes (if it is possible), the terms of the corresponding homogeneous part of degree $n$, obtaining a sequence of maps of the form

$$
F^{(n)}(\mathbf{y})=L \mathbf{y}+F_{n+1}^{(n)}(\mathbf{y})+O\left(|\mathbf{y}|^{n+2}\right),
$$

where $L \mathbf{y}:=F_{1}^{(1)}(\mathbf{y})=D F^{(1)}(\mathbf{0}) \mathbf{y}$.

For instance, the transformation

$$
\mathbf{x}=\Phi_{3}(\mathbf{y}):=\mathbf{y}+\phi_{3}(\mathbf{y})
$$

with $\phi_{3} \in \mathscr{H}_{3}$, conjugates the map $F^{(2)}$ with a new map $F^{(3)}$, via

$$
F^{(2)}\left(\Phi_{3}\right)=\Phi_{3}\left(F^{(3)}\right) .
$$


From the above equation, it can be easily seen that the term $F_{3}^{(3)}=L \phi_{3}(\mathbf{y})-$ $\phi_{3}(L \mathbf{y})+F_{3}^{(2)}(\mathbf{y})$. In other words,

$$
F^{(3)}(\mathbf{y})=L \mathbf{y}+L \phi_{3}(\mathbf{y})-\phi_{3}(L \mathbf{y})+F_{3}^{(2)}(\mathbf{y})+O\left(|\mathbf{y}|^{4}\right) .
$$

If $\phi_{3}(\mathbf{y})$ can be chosen in such a way that

$$
M_{L}\left(\phi_{3}(\mathbf{y})\right):=L \phi_{3}(\mathbf{y})-\phi_{3}(L \mathbf{y})=-F_{3}^{(2)}(\mathbf{y}),
$$

then the order-3 terms of $F^{(3)}$ can be removed and it is transformed into

$$
F^{(3)}(\mathbf{y})=L \mathbf{y}+O\left(|\mathbf{y}|^{4}\right) .
$$

The vectorial equation (16), whose unknowns are the coefficients of $\phi_{3}$, is called the order 3 homological equation associated with $L$, and the existence of solutions of it, is the necessary and sufficient condition to be able to remove the homogeneous terms of degree 3 .

If there is any obstruction to solve the homological equation (16), this can be interpreted as an obstruction in the linearization process. These obstructions are polynomial expressions in the coefficients of $F^{(2)}$, that must vanish in order to continue with the linearization process. The equations obtained by equaling these polynomial expressions to zero are called periodicity conditions; see [13].

For instance, when $L=\operatorname{diag}(\alpha, 1 / \alpha)$, there are no obstructions for the order 2 homological equation. But there appear some ones in the order 3 homological equation. The associated periodicity conditions in this case, are given by

$$
f_{2,1}^{(2)}=0 \text { and } g_{1,2}^{(2)}=0 .
$$

Using the above method, and computing the explicit expressions of equations (17), we prove:

Theorem 23 ([13]) Consider a smooth complex map of the form

$$
F(x, y)=\left(\alpha x+\sum_{i+j \geq 2} f_{i, j} x^{i} y^{j}, \frac{1}{\alpha} y+\sum_{i+j \geq 2} g_{i, j} x^{i} y^{j}\right),
$$

where $\alpha$ is a primitive $p$-root of unity, $p \geq 5$. Then the conditions

$$
\begin{aligned}
& \left(f_{2,1}+f_{1,1} g_{1,1}\right) \alpha^{4}-f_{1,1}\left(2 f_{2,0}-g_{1,1}\right) \alpha^{3}+ \\
& \left(2 g_{2,0} f_{0,2}-f_{1,1} f_{2,0}+f_{1,1} g_{1,1}\right) \alpha^{2}-\left(f_{2,1}+f_{1,1} f_{2,0}\right) \alpha+f_{1,1} f_{2,0}=0, \\
& g_{0,2} g_{1,1} \alpha^{4}-\left(g_{1,2}+g_{0,2} g_{1,1}\right) \alpha^{3}+\left(f_{1,1} g_{1,1}+2 g_{2,0} f_{0,2}-g_{0,2} g_{1,1}\right) \alpha^{2} \\
& +g_{1,1}\left(-2 g_{0,2}+f_{1,1}\right) \alpha+f_{1,1} g_{1,1}+g_{1,2}=0
\end{aligned}
$$


are necessary for $F$ to be p-periodic.

\subsection{An application of Theorem 23}

As an application of an extension of Theorem 23 presented in [13], where an additional third condition is given, we study the global periodicity for the 2-periodic Lyness recurrence

$$
x_{n+2}=\frac{a_{n}+x_{n+1}}{x_{n}}, \quad \text { where } \quad a_{n}=\left\{\begin{array}{l}
a \text { for } n=2 \ell+1, \\
b \text { for } n=2 \ell,
\end{array}\right.
$$

and $a, b \in \mathbb{C}$. We obtain:

Theorem 24 ([13]) The only globally periodic recurrences in (19) are:

(i) The cases $a=b=0$ which is 6-periodic, and $a=b=1$, which is 5-periodic.

(ii) The cases $a=(-1 \pm i \sqrt{3}) / 2$ and $b=\bar{a}=1 / a$, which is 10-periodic.

Notice that the cases given in (i) correspond to the well-known autonomous globally periodic Lyness recurrences given in Example 1. The ones corresponding to (ii) are new non-autonomous globally periodic recurrences.

Observe that the sequence $\left\{x_{n}\right\}$ can be reobtained as

$$
\left(x_{1}, x_{2}\right) \stackrel{G_{a}}{\longrightarrow}\left(x_{2}, x_{3}\right) \stackrel{G_{b}}{\longrightarrow}\left(x_{3}, x_{4}\right) \stackrel{G_{a}}{\longrightarrow}\left(x_{4}, x_{5}\right) \stackrel{G_{b}}{\longrightarrow}\left(x_{5}, x_{6}\right) \stackrel{G_{a}}{\longrightarrow} \cdots
$$

where $G_{\alpha}(x, y)=(y,(\alpha+y) / x)$, with $\alpha \in\{a, b\}$. So the behavior of (19) is determined by the dynamical system generated by the map:

$$
F(x, y):=G_{b} \circ G_{a}(x, y)=\left(\frac{a+y}{x}, \frac{a+b x+y}{x y}\right)
$$

which always has some fixed point. In [13], the extension of Theorem 23 is applied to $F$ for proving Theorem 24 ,

Acknowledgements The authors are partially supported by Spanish Ministry of Economy and Competitiveness through grants MTM2008-03437 (first and second authors); DPI2011-25822 (third author); and MTM2011-26995-C02-01 (fourth author). Both CoDALab and GSD-UAB groups are supported by Generalitat de Catalunya through the SGR program.

\section{References}

1. Arrowsmith D.K., Place C.M.: An introduction to dynamical systems. Cambridge University Press, Cambridge, (1990) 
2. Bellon M.P.: Algebraic Entropy of Birational Maps with Invariant Curves. Lett. Math. Phys. 50, 79-90 (1999)

3. Balibrea F., Linero-Bas A.: Some new results and open problems on periodicity of difference equations. In: Iteration theory (ECIT 04), Grazer Math. Ber. 350, 15-38 (2006)

4. Bing, R.H.: Inequivalent Families of Periodic Homeomorphisms of E3. Annals of Mathematics. 56, 354-362 (1952)

5. Bing, R.H.: A homeomorphism between the 3-sphere and the sum of two solid horned spheres. Annals of Mathematics. 80, 78-93 (1964)

6. Bedford E., Kim K.: Periodicities in linear fractional recurrences: degree growth of birational surface maps. Michigan Math. J. 54, 647-670, (2006)

7. Bedford E., Kim K.: Linear fractional recurrences: periodicities and integrability. Ann. Fac. Sci. Toulouse Math. 20, 33-56 (2011).

8. Bochner, S.: Compact groups of differentiable transformations. Annals of Mathematics. 46, 372-381 (1945)

9. Chow, S.-N.; Li, C.; Wang, D.: Normal forms and bifurcation of planar vector fields. Cambridge University Press, Cambridge, (1994)

10. Cima A., Gasull A., Mañosa V.: Global periodicity and complete integrability of discrete dynamical systems. J. Difference Equations and Appl. 12, 697-716 (2006)

11. Cima A., Gasull A., Mañosa V.: Studying discrete dynamical systems through differential equations. J. Differential Equations 244, 630-648 (2008)

12. Cima A., Gasull A., Mañosa V.: Some properties of the k-dimensional Lyness' map. J. Physics A: Mathematical \& Theoretical, 41, 285205 (2008)

13. Cima A., Gasull A., Mañosa V.: Global periodicity conditions for maps and recurrences via Normal Forms. arXiv:1205.0923v1 [math.DS] (2012)

14. Cima A., Gasull A., Mañosas F.: On periodicity rational difference equations of order $k$. J. Difference Equations and Appl. 10, 549-559 (2004)

15. Cima A., Gasull A., Mañosas F.: New periodic recurrences with applications. J. Math. Anal. Appl. 382, 418-425 (2011)

16. Cima A., Gasull A., Mañosas F.: Global linearization of periodic difference equations. Discrete Contin. Dyn. Syst. 32, 1575-1595 (2012)

17. Cima A., Gasull A., Mañosas F.: Simple examples of planar involutions with non-global Montgomery-Bochner linearizations. Appl. Math. Lett. 25, 2086-2088 (2012)

18. Cima A., Gasull A., Mañosas F.: On Coxeter recurrences. J. Difference Equations and Appl. 18, 1457-1465 (2012).

19. Constantin A., Kolev B.: The theorem of Kerékjártó on periodic homeomorphisms of the disc and the sphere. Enseign. Math. 40, 373-413 (1994)

20. Csörnyei, M., Laczkovich, M.: Some periodic and non-periodic recursions. Monatshefte für Mathematik 132, 215-236 (2001)

21. Conder M.: Hurwitz groups with given centre. Bull. London Math. Soc. 34, 725-728 (2002)

22. Conder M.: An update on Hurwitz groups. Groups Complex. Cryptol. 2, 35-49 (2010)

23. Coxeter, H.S.M.: Frieze patterns. Acta Arith. 18, 297-310 (1971)

24. Diller J., Favre C.: Dynamics of bimeromorphic maps of surfaces. Amer. J. Math. 123, 1135$1169(2001)$

25. Epstein, D.B.A.: Periodic flows on three-manifolds. Annals of Mathematics. 95, 66-82 (1972)

26. Farr, W.W., Li, C., Labouriau, I.S., Langford, W.F.: Degenerate Hopf bifurcation formulas and Hilbert's 16th problem. SIAM J. Math. Anal. 20, 13-30 (1989)

27. Guckenheimer J., Holmes P.: Nonlinear Oscillations, Dynamical Systems, and Bifurcations of Vector Fields. Springer, New York (1983)

28. Grove E.A., Ladas G.: Periodicities in Nonlinear Difference Equations Equations. Advances in discrete Math. and Appl, vol. 4. Chapman \& Hall/CRC Press, Boca Raton FL (2005)

29. Guillemin V., Pollack A.: Differential Topology. Prentice Hall. Englewood Cliffs, New Jersey (1974)

30. Haggar F., Byrnes G.B., Quispel G.R.W., Capel H.W.: $k$-integrals and $k$-Lie symmetries in discrete dynamical systems. Physica A 233, 379-394 (1996) 
31. Hassard B., Kazarinoff N.D., Wan Y.H.: Theory and applications of the Hopf Bifurcations. Cambridge University Press, Cambridge (1980)

32. Hayes R., Kwasik S., Mast J., Schultz R.: Periodic maps $\mathbb{R}^{7}$ without fixed points. Math. Proc. Cambridge Philos. Soc. 132, 131-136 (2002)

33. Jogia D., Roberts J. A. G., Vivaldi F.: An algebraic geometric approach to integrable maps of the plane. J. Physics A: Mathematical \& General 39, 1133-1149 (2006)

34. Kister, J.M.: Differentiable periodic actions on $E^{8}$ without fixed points. Amer. J. Math. 85, 316-319 (1963)

35. Kurshan, R.P., Gopinath B.: Recursively generated periodic sequences, Canad. J. Math. 26, 1356-1371 (1974)

36. Linero-Bas A.: Some results on periodicity of difference equations. In: Liz, E., Mañosa V. (eds.) Proceedings of the Workshop Future Directions in Difference Equations, pp. 121-143. Universidade de Vigo, Vigo (2011)

37. Maksymenko S. I.: Period functions for $\mathscr{C}^{0}$ - and $\mathscr{C}^{1}$-flows. Ukrainian Math. J. 62, 1109-1125 (2010)

38. Montgomery D.: Pointwise Periodic Homeomorphisms. Amer. J. Math. 59, 118-120 (1937)

39. Montgomery D., Zippin L.: Topological Transformation Groups. Interscience, New York, (1955)

40. Peralta-Salas D.: Private Communication (2009)

41. Reeb G.: Sur certaines proprietes topologiques des varietes feuilletees. Actual. Sci. Ind. 1183, Hermann, Paris (1952)

42. Rubió-Massegú J.: On the global periodicity of discrete dynamical systems and application to rational difference equations. J. Math. Anal. Appl. 343, 182-189 (2008)

43. Rubió-Massegú J., Mañosa V.: Normal forms for rational difference equations with applications to the global periodicity problem. J. Math. Anal. Appl. 332, 896-918 (2007)

44. Sullivan D.: A counterexample to the periodic orbit conjecture. Inst. Hautes Etudes Sci. Publ. Math. 46, 5-14 (1976)

45. Pires B., Teixeira M.A.: On global linearization of planar involutions, arxiv:1105.4890v1 (2011)

46. Ochiai T., Nacher J.C.: Inversible Max-Plus algebras and integrable systems. Journal of Mathematical Physics 46, 063507 (2005)

47. Vogt E.: A periodic flow with infinite Epstein hierarchy. Manuscr. Math. 22, 403-412 (1977)

48. Wiggins S.: Introduction to Applied Nonlinear Dynamical Systems and Chaos. Springer, New York, (1990) 